\title{
Challenge for open accessed journals
}

\author{
Michael W Roberts \\ Department of Paediatric Dentistry, University of North Carolina Adams School of Dentistry, USA
}

Several years ago, I authored a short opinion paper titled "Formulating the research question, conducting the investigation and publishing the results" [1]. The paper focused on the importance of research to continue to provide the dental profession with current information necessary to improve the standard of patient care. However, as challenging as obtaining funding for such research, the challenge to get it published can be equally daunting. The number of traditional peer-reviewed journals have been over-whelmed with manuscript submissions for publication consideration and they often reject up to $90 \%$ of submissions. This dilemma had created a bottleneck for dissemination of new information and science. Responding to a recognized need, open access journals arose.

These new journals have been widely accepted and embraced but they often vary in the intensity of peer review prior to publication. One reason for this slight is that the number of open access journals have mushroomed and the competition to attract investigators to consider their journal for submission of their work can be intense. Investigators need to have their work published but they also want the journal to have a good reputation. To achieve this goal, the journal must recruit and engage dedicated competent reviewers who are committed to improving the value of the publication.

In addition, the journal publishers must do whatever is required to have their journal recognized by leading indexing services (e.g. Pub Med). This not only improves the reputation of the journal but also increases its access to other investigators or readers and makes the publication more attractive to researchers. This is an acknowledged challenge but is very important to success.

The fee to publish in an open-access journal can also be a restricting factor. The publication fees can often exceed \$1000 US depending upon the length of the manuscript and the number of figures or tables. This cost can be an impediment to many investigators especially those in countries with restrictive economies. I recognize that journal publications not financially supported by a professional organization is a business enterprise and covering costs and producing a profit are important to the survival of the journal. However, the open access publishers should attempt to reach out to manufacturers or foundations to obtain support of their mission and product in order to reduce the financial impediment to the manuscript authors. The authors have already had to garner financial support for their research, conduct the research and prepare the manuscript. It seems counter initiative that they offer their work to a journal for publication consideration but also are expected to pay for the privilege.

Access to journals to published research and clinical reports is essential to continuing to move our profession forward. Hopefully, this need will stimulate creative solutions to the existing problems.

\section{References}

1. Roberts MW (2016) Formulating the research question, conducting the investigation and publishing the result. Int J Dent Oral Health 2.
Copyright: (C)2019 Roberts MW. This is an open-access article distributed under the terms of the Creative Commons Attribution License, which permits unrestricted use, distribution, and reproduction in any medium, provided the original author and source are credited.
*Correspondence to: Michael W. Roberts, Department of Paediatric Dentistry, University of North Carolina Adams School of Dentistry, USA, E-mail: Mike Roberts@unc.edu

Received: March 03, 2019; Accepted: March 15, 2019; Published: March 20, 2019 